BI-TP 2006/20

CU-TP-1148

\title{
Diffractive dissociation including pomeron loops in zero transverse dimensions
}

\author{
Arif I. Shoshi ${ }^{1, *}$ and Bo-Wen $\mathrm{XiaO}^{2,+}$ \\ ${ }^{1}$ Fakultät für Physik, Universität Bielefeld, D-33501 Bielefeld, Germany \\ ${ }^{2}$ Department of Physics, Columbia University, New York, NY, 10027, USA
}

(Dated: July 7, 2018)

\begin{abstract}
We have recently studied the QCD pomeron loop evolution equations in zero transverse dimensions [1]. Using the techniques developed in [1] together with the AGK cutting rules, we present a calculation of single, double and central diffractive cross sections (for large diffractive masses and large rapidity gaps) in zero transverse dimensions in which all dominant pomeron loop graphs are consistently summed. We find that the diffractive cross sections unitarise at large energies and that they are suppressed by powers of $\alpha_{s}$. Our calculation is expected to expose some of the diffractive physics in hadron-hadron collisions at high energy.

PACS numbers: 13.85-t;13.85.Hd; $11.55 .-\mathrm{m} ; 11.55 . \mathrm{Bq}$
\end{abstract}

*Electronic address: shoshi@physik.uni-bielefeld.de

${ }^{\dagger}$ Electronic address: bowen@phys.columbia.edu 


\section{INTRODUCTION}

QCD evolution equations have been recently established which include pomeron loops (or fluctuations) [2]. The main feature of these equations, as compared to the BalitskyJIMWLK [3, 4] or Kovchegov equations [5], are the violation of the geometrical scaling of the $T$-matrix and a modified energy dependence for the saturation momentum [6]. Recently the effect of fluctuations also on single diffractive dissociation [7] has been studied and compared with previously obtained mean-field-like results [8] at high energies.

In a recent paper [1] we have studied the QCD pomeron loop equations in zero transverse dimensions in which case one can do the calculation of any pomeron loop graph analytically (see also [9, 10, 11, 12, 13, 14, 15, 16]). The pomeron loop equations are a hierarchy of equations which in zero transverse dimensions take the form

$$
\frac{d n^{(k)}}{d y}=k \alpha n^{(k)}+k(k-1) \alpha n^{(k-1)}-k \beta n^{(k+1)},
$$

where $n^{(k)}$ is a normal ordered number operator defined as $\langle\widetilde{n}(\widetilde{n}-1) \cdots(\widetilde{n}-k+1)\rangle$ which represents the expectation value of $k$-pomerons during the evolution. The single terms in eq. (11) have the following physical meaning: $k \alpha n^{(k)}$ is the BFKL growth term ( $\alpha$ here corresponds to $\alpha_{P}-1=\frac{4 \alpha_{s} N_{c}}{\pi} \ln 2$ in the real BFKL equation), $k(k-1) \alpha n^{(k-1)}$ describes fluctuations (pomeron splittings) and $k \beta n^{(k+1)}$ recombinations (pomeron mergings). Eq. (1) is the zero-transverse-dimensional analog of the real QCD equations (see [2]). One can easely show that (11) leads to frame-independent scattering amplitudes when $\beta=\alpha \alpha_{s}^{2}$.

The scattering amplitudes have been calculated in Ref. [1] by treating the recombination terms as small perturbations. In a recent paper together with our collaborators [14] we have shown that at rapidity $Y \gg 1 / \alpha_{s}^{2} \alpha$, the perturbative treatment of the recombination terms becomes inaccurate and needs to be replaced by more complete calculations (This limit is

also indicated in our corrections to the LO result given in Eq. (15) in Ref. [1]). Thus, the results for diffractive cross sections shown in this paper are only reliable up to $Y \approx 1 / \alpha_{s}^{3}$.

In this paper we use the techniques which we have developed in [1] together with the AGK cutting rules [17] to calculate single, double and central diffractive cross sections in zero transverse dimensions (see Fig. 1). We do consider only the case where the diffractive masses, rapidity gaps and the total rapidity are large. All the relevant pomeron graphs are included in the calculations. In four dimensional QCD, the analogous calculations are not 
yet available. So far only the single diffractive scattering in real QCD including pomeron loops has been calculated, however, only in the kinematical region where the diffractive mass is not too large [7]. Thus, our calculation in zero transverse dimensions may give indications about the diffractive mass dependence of the diffractive cross section in four dimensional QCD at very large diffractive masses. We expect the energy dependence of the QCD high energy diffractive scattering would bear some resemblances of our result.

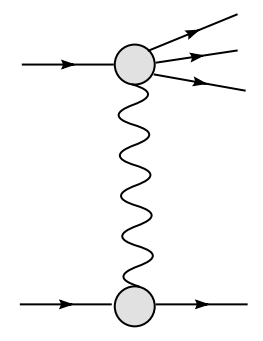

$A$

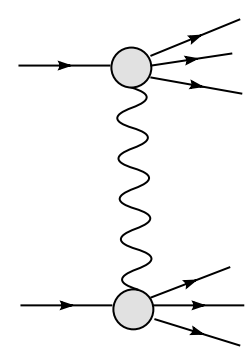

$B$

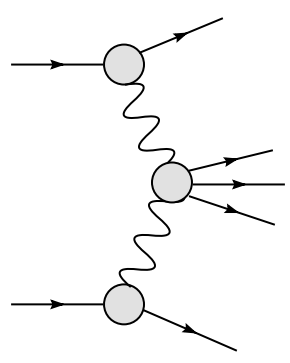

C

FIG. 1: Typical diffractive dissociation graphs: Single diffractive scattering (A), double diffractive scattering (B), central diffractive scattering (C).

A longstanding problem has been accompanying diffractive dissociation: The lowest order pomeron contribution to diffractive dissociation disagrees with the Fermilab data (see e.g., [18]) and it violates unitarity at very high energies. There have been several attempts to solve this problem by considering the renormalization of the pomeron flux [18], a larger impact parameter [19] or a smaller survival probability [20] at high energies. In this work we show that single, double and central diffractive cross sections would naturally fulfill unitarity limits when multiple pomeron exchanges are taken into account in addition to the lowest order pomeron exchange.

Furthermore we do find that the differential diffractive cross sections (differential with respect to the masses) are suppressed by powers of $\alpha_{s}$. We will show that this suppression is characteristic for differential diffractive cross sections and emerges from pomeron splittings/mergings in the t-channel which are needed in order to calculate the differential diffractive cross sections.

The paper is organized as follows: We do start with an illustration of the AGK cutting rules, then go over to the single diffractive cross section, which is then followed by double 
diffractive and central diffractive cross sections.

\section{THE AGK CUTTING RULES}

The AGK cutting rules [17] (see also [9, 21]) represent the generalization of the optical theorem for the case of multiple pomeron exchange. Although AGK cutting rules have not been proven for QCD, they have been discussed and used in several publications (see Refs. [22, 23, 24]) . We will show below how the AGK cutting rules lead to expectable results for particle-particle and particle-nucleus results also within this QCD model, and then use them to also calculate diffractive scattering processes.

To illustrate the AGK cutting rules, let us consider for instance the total cross section for particle-particle scattering,

$$
\sigma_{\text {tot }}=2 \sum_{n=1}^{\infty}(-1)^{n-1} F^{n},
$$

where $F^{n}$ is the amplitude for the exchange of $n$ pomerons. According to the AGK cutting rules, $\sigma_{t o t}$ is related to the elastic cross section $\sigma_{0}$ and inelastic cross section $\sigma_{i n}$,

$$
\sigma_{t o t}=\sigma_{0}+\sigma_{i n}
$$

which are given by

$$
\sigma_{0}=\sum_{n=2}^{\infty} F_{0}^{n}
$$

and $^{1}$

$$
\sigma_{i n}=\sum_{k=1}^{\infty} \sigma_{k}=\sum_{k=1}^{\infty} \sum_{n=k}^{\infty} F_{k}^{n} .
$$

In the above equations $F_{0}^{n}$ represents the $n$-pomeron exchange graph where no pomeron is cut (elastic cut) as shown in Fig. $2 \mathrm{~B}$ and $F_{k}^{n}$ is the $n$-pomeron exchange graph with $k$ pomerons being simultaneously cut (inelastic cut) as shown in Fig. 2A (cut pomerons are marked by crosses). In the next sections we will introduce also the diffractive cut as shown

\footnotetext{
${ }^{1}$ In eq. (4) $n$ starts from 2 because there have to be at least two pomerons, one pomeron on either side of the elastic cut. One side of the cut can be viewed as initial state of the scattering while the other side can be understood as the final state.
} 

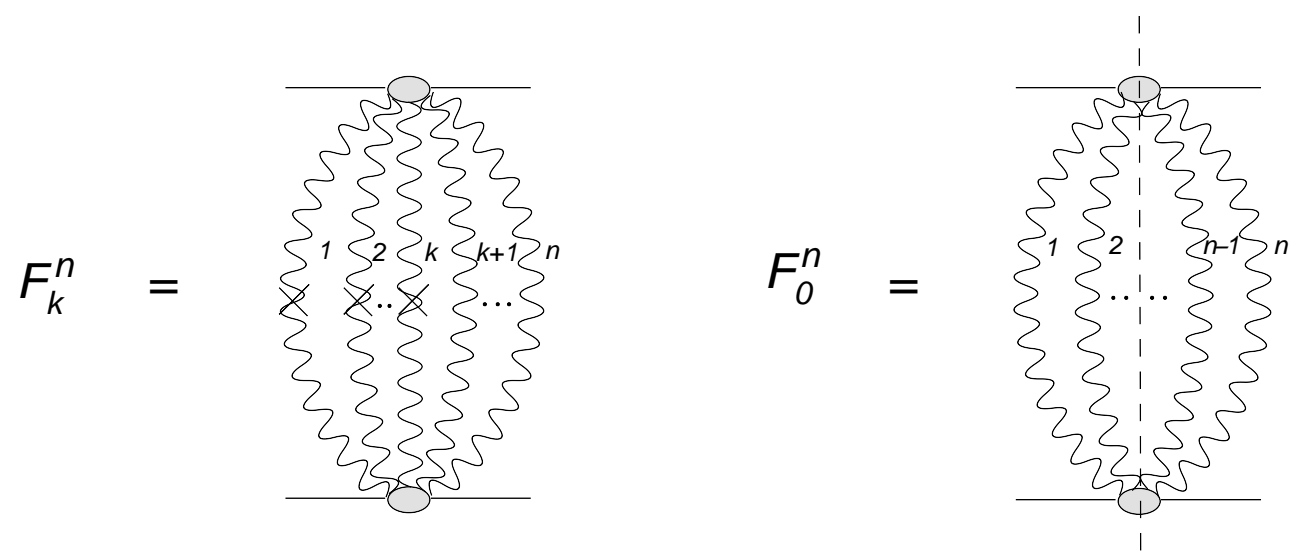

FIG. 2: $n$-pomeron exchange graphs: In graph $\mathrm{A} k$ of the $n$-pomerons are cut (inelastic cut) and in graph B none of the pomerons is cut (elastic cut).

for example in Fig. 3A where only a part of a pomeron is cut. The diffractive cut generates diffractive (dissociation) cross sections while the inelastic cut (cut of an entire pomeron) leads to a uniform production of final state particles in rapidity.

For convenience, let us summarize the AGK cutting rules, which allow us to calculate $F_{0}^{n}$ and $F_{k}^{n}$ and the diffractive cross sections in the next sections:

- No matter how many pomerons are cut, there is always one and only one cut which indicates the separation between the initial and final states of the scattering;

- Each cut-pomeron gives an extra factor of $(-2)$, which can be understood as a result of the discontinuity of the pomeron amplitude;

- Each un-cut pomeron obtains an extra factor of 2 since it can be placed on either side of the cut.

Thus, applying the above AGK cutting rules on Fig. 2, we obtain

$$
F_{0}^{n}=(-1)^{n} 2\left(2^{n-1}-1\right) F^{n}
$$

and

$$
F_{k}^{n}=(-1)^{n}(-2)^{k}(2)^{n-k} C_{n}^{k} F^{n}
$$


where $C_{n}^{k}=n ! /[k !(n-k) !]$ is the number of selections of $k$ pomerons out of $n$ pomerons. It is easy to verify Eq. (3) by substituting the above expressions for $F_{0}^{n}$ and $F_{k}^{n}$ in Eq. (44) and Eq. (5), respectively.

To gain confidence in the AGK cutting rules, let us show that the cross sections obtained using the AGK cutting rules and the pomeron calculus do agree with known unitarity limits at high energies. For particle-particle scattering the amplitude for $n$-pomeron exchanges at leading order [1] is $F^{n}=n !\left(\alpha_{s}^{2} e^{\alpha Y}\right)^{n}$ (the factor $(-1)^{n}$ has already been taken into account in Eqs.(24, (20)). Inserting this amplitude in Eqs.(2],4],5), one finds $\sigma_{\text {tot }} \simeq 2, \sigma_{\text {inel }} \simeq 1$ and $\sigma_{0} \simeq 1$ in large $Y$ limit, which is in agreement with the black disk limit. For particle-nucleus scattering, now with $F^{n}=\left(\alpha_{s}^{2} e^{\alpha Y} L\right)^{n}$, where $L$ is the number of hadrons in the nucleus and $\alpha^{2} L \gg 1$, it is straightforward to obtain the following results for the total, elastic (diffractive) and inelastic cross sections,

$$
\begin{aligned}
\sigma_{t o t} & =\frac{2 \alpha_{s}^{2} e^{\alpha Y} L}{1+\alpha_{s}^{2} e^{\alpha Y} L} \\
\sigma_{0} & =\frac{2\left(\alpha_{s}^{2} e^{\alpha Y} L\right)^{2}}{\left(1+\alpha_{s}^{2} e^{\alpha Y} L\right)\left(1+2 \alpha_{s}^{2} e^{\alpha Y} L\right)} \\
\sigma_{i n} & =\frac{2 \alpha_{s}^{2} e^{\alpha Y} L}{1+2 \alpha_{s}^{2} e^{\alpha Y} L}
\end{aligned}
$$

which do as well respect unitarity limits at very high rapidity.

\section{SINGLE DIFFRACTIVE SCATTERING}

The single diffractive process, $p+p \rightarrow p+X$, as sketched in Fig. 1, is a process where one of the $p$-particles breaks up into a "diffractive state" $X$ which is separated by a rapidity gap from the $p$-particle in the final state which remains intact. In the language of pomerons, the single diffractive production is generated by a diffractive cut of a multiple pomeron exchange graph as shown in Fig. 3A (lowest order graph) and Fig. 3B (higher order graph). The appropriate variables to describe the single diffractive scattering process are the rapidities

$Y=\ln \frac{s}{m_{p}^{2}}$ and $Y_{0}=\ln \frac{M_{X}^{2}}{m_{p}^{2}}$, where $s$ is the square of the center of mass energy, $M_{X}$ is the diffractive mass, and $m_{p}$ is the rest mass of the particle $p$. In this picture, the rapidity gap $Y-Y_{0}$ is easily understood since there is no cut through any of the pomerons in this rapidity window.

We calculate the single diffractive cross section for the case where $Y, Y_{0}$ and $Y-Y_{0}$ 


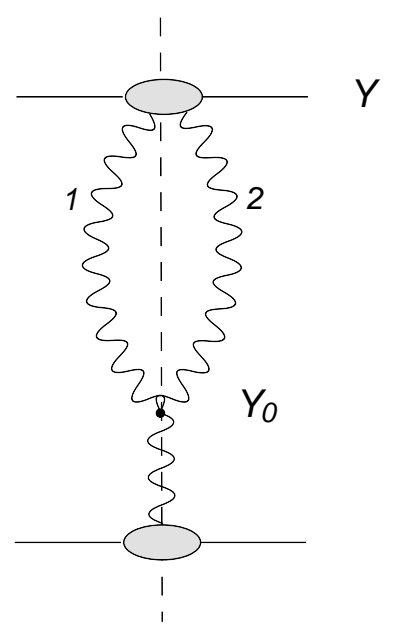

$A$

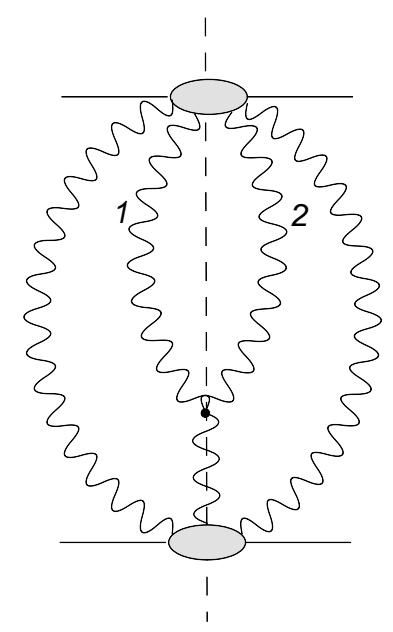

$B$

FIG. 3: Diffractive cut of the multiple pomeron exchange graphs which generate single diffractive dissociation: Diagram A is the lowest order graph and diagram B is a higher order graph.

are large. In this kinematical window the dominant graphs are those shown in Fig. 3, The leading order contribution of the dominant graphs is calculated in this work. (The subdominant graphs which we neglect are those where pomeron loops are formed in the diffractive region $0<y<Y_{0}$ or in the rapidity gap region $Y_{0}<y<Y$. The loops in these windows are smaller as compared to the dominant loops stretching over the whole rapidity $Y$ and give therefore small corrections, proportional to $\exp \left(-\alpha Y_{0}\right)$ or $\exp \left[-\alpha\left(Y-Y_{0}\right)\right]$, in comparison to the main result coming from the large loops. In addition there are also other subleading contributions neglected here which are $\alpha_{s}$ suppressed as compared to the main result which are discussed in detail in Ref. [1].).

According to the topology of Fig. 3 and the AGK cutting rules summarized above, we get for the differential single diffractive cross section ${ }^{2}$

$$
\frac{d \sigma_{S D}\left(Y, Y_{0}\right)}{d Y_{0}}=\sum_{n=2}^{\infty}(-1)^{n} 2^{n-1} F^{n}\left(Y, Y_{0}\right)
$$

where the factor $2^{n-1}$ indicates the number of different diagrams when the diffractive cut is made and $F^{n}$ is the uncut single diffractive amplitude for the case of $n$-pomeron exchange.

\footnotetext{
${ }^{2}$ We have $d \sigma_{S D} / d Y_{0}$ instead of $\sigma_{S D}$ in Eq. (11) since we have considered the production of a particular diffractive mass $M_{X}$ (or $\left.Y_{0}=\ln \left(M_{X}^{2} / m_{p}^{2}\right)\right)$.
} 
We extract $F^{n}$ from the $T$-matrix for the uncut graphs. The latter is calculated within the zero transverse dimensional model [1] which we have recently developed. In the limit of $\exp \left(\alpha Y_{0}\right) \gg 1$ and $\exp \left[\alpha\left(Y-Y_{0}\right)\right] \gg 1$, the dominant term is the one where $k$ pomeron splittings occur at $Y=0$,

$$
n_{k}^{(k+1)}(Y) \simeq(k+1) ! e^{(k+1) \alpha Y}
$$

the last pomeron splitting occurs at $Y_{0}$,

$$
\begin{aligned}
n_{k}^{(k+2)}\left(Y, Y_{0}\right) & \simeq(k+2)(k+1) \int_{0}^{Y} \alpha d y n_{k}^{(k+1)}(y) e^{(k+2) \alpha(Y-y)} \delta\left(y-Y_{0}\right) \\
& \simeq \alpha(k+1)(k+2) ! e^{(k+2) \alpha Y-\alpha Y_{0}}
\end{aligned}
$$

and all the $k+1$ pomeron mergings occur at $Y$

$$
n_{k}^{(1)}\left(Y, Y_{0}\right) \simeq(-1)^{k+1} \alpha(k+1)(k+2) ! x^{k} e^{2 \alpha Y-\alpha Y_{0}}
$$

where $x=\alpha_{s}^{2} e^{\alpha Y}$ and $T_{k}^{(1)}\left(Y, Y_{0}\right)=\alpha_{s}^{2} n_{k}^{(1)}\left(Y, Y_{0}\right)$. Now, from $T_{k}^{(1)}\left(Y, Y_{0}\right)=$ $(-1)^{k} \alpha(k+1)(k+2) ! x^{k+2} e^{-\alpha Y_{0}}$, we extract $F^{n}=\alpha(n-1) n ! x^{n} e^{-\alpha Y_{0}}$ by using $F^{n}=$ $(-1)^{n} T_{n-2}^{(1)}$, which inserted into the diffractive cross section given in Eq. (11) yields

$$
\begin{aligned}
\frac{d \sigma_{S D}\left(Y, Y_{0}\right)}{d Y_{0}} & \simeq \alpha e^{-\alpha Y_{0}} \sum_{n=2}^{\infty}(-1)^{n} 2^{n-1}(n-1) n ! x^{n} \\
& =-\frac{1}{2} \alpha e^{-\alpha Y_{0}} z^{2} \frac{d^{2}}{d z^{2}}\left[\Gamma\left(0, \frac{1}{z}\right) e^{\frac{1}{z}}\right]
\end{aligned}
$$

where we have used the definition $z=2 \alpha_{s}^{2} e^{\alpha Y}$. The first term of the sum in Eq. (14) reproduces the energy dependence of the first order Regge prediction for single diffractive processes, and other terms in the summation are contributions of higher order dominant graphs. In the high energy limit, when the diffractive mass (or $Y_{0}$ ) and the rapidity gap $\left(Y-Y_{0}\right)$ are kept large, we find

$$
\frac{d \sigma_{S D}\left(Y, Y_{0}\right)}{d Y_{0}} \simeq \frac{1}{2} \alpha e^{-\alpha Y_{0}}\left[1+\frac{1+2 \gamma_{E}-2 \ln \left(2 \alpha_{s}^{2} e^{\alpha Y}\right)}{2 \alpha_{s}^{2} e^{\alpha Y}}\right],
$$

or, in terms of the diffractive mass,

$$
M_{x}^{2} \frac{d \sigma_{S D}\left(s, M_{x}^{2}\right)}{d M_{x}^{2}} \simeq \frac{1}{2} \alpha\left(\frac{m_{p}^{2}}{M_{x}^{2}}\right)^{\alpha} .
$$

where $\gamma_{E}=0.577$ is the Euler constant. 
Here, we would like to comment that Eq. (16) is approximately true when $\alpha Y_{0}$ and $\alpha\left(Y-Y_{0}\right)$ are relatively large, where we find the result $\frac{1}{2} \alpha e^{-\alpha Y_{0}}$. The expectation that all inelastic diffraction vanishes for $Y \rightarrow \infty$ [7] can not be shown in our model since our model does not apply for $Y \gg 1 / \alpha_{s}^{3}$. The origin of our result is obvious: There are $(k+1)$ pomerons in the region $0<y<Y_{0}$. At $Y_{0}$ another pomeron is produced through pomeron splitting enforced by the delta-function in Eq. (12), see also Fig. 3, adding a contribution $\alpha e^{\alpha\left(Y-Y_{0}\right)}$ to the $(k+1)$ pomerons of length $Y$. This explains the appearance of $\alpha e^{-\alpha Y_{0}}$. The remaining $e^{\alpha Y}$-dependence together with the other pomerons of the same length $Y$ do give a sum over all pomeron exchanges which naturally unitarizes the single diffractive cross section at large $Y$. The factor of $1 / 2$ in (16) is a combinatorial factor emerging after the diffractive cut is made.

Another phenomenon not observed so far elsewhere is that the single diffractive cross section in (16) is suppressed by a factor of $\alpha$. This factor appears through the pomeron splitting at $Y_{0}$. A suppression by powers of $\alpha$ is characteristic for differential diffractive cross sections because pomeron splittings in the $t$-channel (see Figs. 3,4,5) are needed in order to calculate them. In the next sections we show how also double and central diffractive cross sections are suppressed by powers of $\alpha$.

It is tempting to integrate Eq. (16) over $Y_{0}$ from 0 to $Y$ to obtain a formula for $\sigma_{S D}(Y)$. However, this wouldn't be right since Eq. (16) is not valid for small values of diffractive masses and rapidity gaps. When diffractive masses or rapidity gaps are small, many other graphs have to be considered besides the dominant graphs shown in Fig. 3. In this case the resummation of all order graphs is no longer under control within our formalism.

In Ref. [7] the (differential) single diffractive cross section in four dimensions including pomeron loops has been calculated. However, the calculation is only applicable in the region where the diffractive mass $Y_{0}$ is small, $Y_{0} \ll \pi /\left(\alpha_{s} N_{c}\right) \ln \left(\pi^{2} / \alpha_{s}^{2}\right)$. On the other hand, our result applies when the diffractive mass is large, $\exp \left[\alpha Y_{0}\right] \gg 1$. It would be interesting to see whether our result remains valid also in four dimensional QCD.

\section{DOUBLE DIFFRACTIVE SCATTERING}

The double diffractive scattering process, $p+p \rightarrow X+X^{\prime}$ (see Fig. 1B), is generated by the three topologically different types of diffractively cut multiple pomeron exchanges as shown 

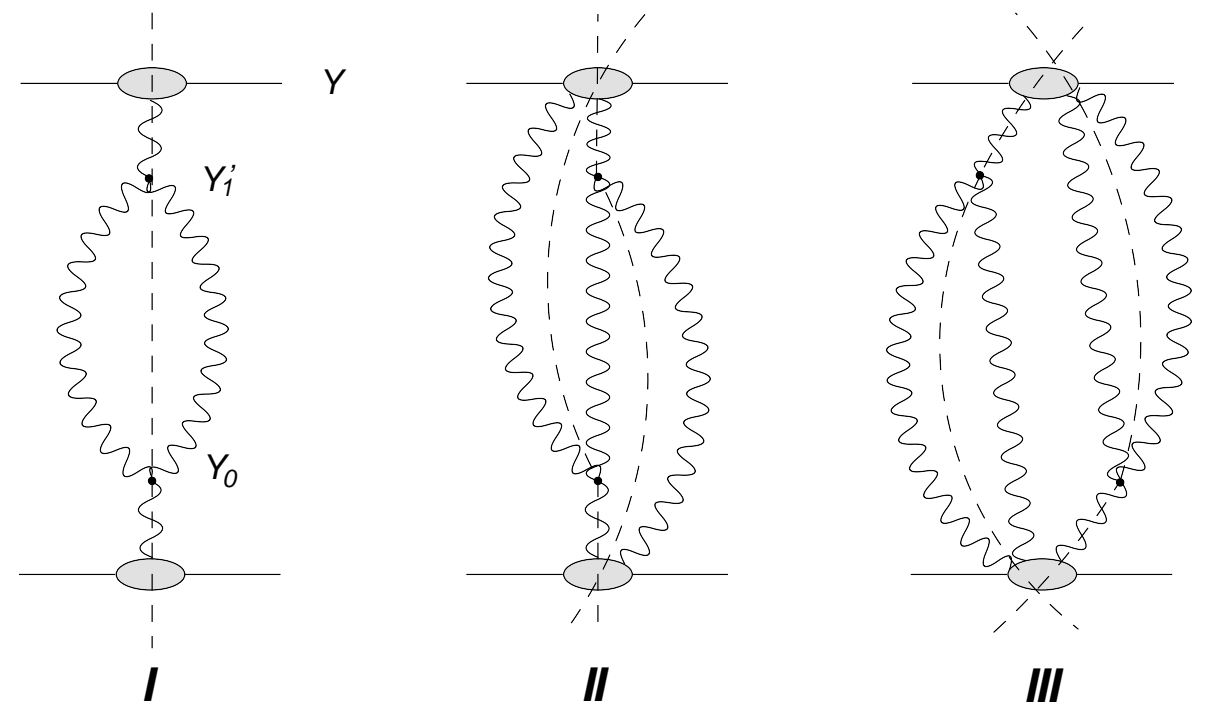

FIG. 4: Three topologically different types of the diffractively cut multiple pomeron exchange graphs which generate double diffractive dissociation. Diagram I (type I) is the lowest order graph; Diagram II (type II) is a higher order graph which requires at least 3 pomerons exchanged; Diagram III (type III) is another higher order graph which needs at least 4 pomerons exchanged.

in the lowest order in Fig. 4. We use below the variables $Y_{0}=\ln \frac{M_{X}^{2}}{m_{p}^{2}}$ and $Y_{1}=Y-Y_{1}^{\prime}=\ln \frac{M_{X}^{\prime 2}}{m_{p}^{2}}$, where $M_{X}$ and $M_{X}^{\prime}$ are the diffractive masses, to express the double diffractive cross section. The rapidity gap between the diffractive states $X$ and $X^{\prime}$ is $Y-Y_{0}-Y_{1}$.

Let us first compute the dominant contribution of the un-cut amplitude for the case of multiple pomeron exchange with one pomeron splitting at $Y_{0}$ and one pomeron merging at $Y_{1}$. Further we consider the case where $Y_{0}$ and $Y_{1}$ are kept large. The result after the last pomeron splitting at $Y_{0}$ which is given by Eq. (12), when followed by a pomeron merging at $Y_{1}^{\prime}$, gives

$$
\begin{aligned}
n_{k}^{(k+1)}\left(Y_{1}^{\prime}, Y_{0}, Y\right) & \simeq-\alpha_{s}^{2}(k+1) \int_{0}^{Y} \alpha d y n_{k}^{(k+2)}(y) e^{(k+1) \alpha(Y-y)} \delta\left(y-Y_{1}^{\prime}\right), \\
& =\left(-\alpha_{s}^{2}\right)(k+2) !(k+1)^{2} \alpha^{2} e^{(k+1) \alpha Y+\alpha Y_{1}^{\prime}-\alpha Y_{0}}
\end{aligned}
$$

and after $k$ more pomeron mergings at rapidity $Y$ (see [1] ), one ends up with $T_{k}^{(1)}\left(Y, Y_{1}, Y_{0}\right) \simeq$ $(-1)^{k+1} \alpha^{2}(k+1)^{2}(k+2) ! x^{k+2} e^{-\alpha Y_{1}-\alpha Y_{0}}$, where $Y_{1}=Y-Y_{1}^{\prime}$.

The resulting $F_{D D}^{n}=\alpha^{2}(n-1)^{2} n ! x^{n} e^{-\alpha Y_{0}-\alpha Y_{1}}$ has the following interpretation: There are $(n-1)^{2}$ different graphs in the $n$-pomeron exchange case, each of them contributing by the same amplitude $\alpha^{2} n ! x^{n} e^{-\alpha Y_{0}-\alpha Y_{1}}$. Among these $(n-1)^{2}$ pomeron exchange graphs, 
there are three topologically different types when the diffractive cut is made (e.g., see Fig. 4). In the $n$-pomeron exchange case, it is easy to find that there are $N_{I}^{(n)}=(n-1)$ diagrams of the first type, $N_{I I}^{(n)}=2(n-2)$ diagrams of the second type and $N_{I I I}^{(n)}=(n-2)(n-3)$ diagrams of the third type. (Evidently, $N_{I}^{(n)}+N_{I I}^{(n)}+N_{I I I}^{(n)}=(n-1)^{2}$.) When the cut is made, the number of diagrams of type I becomes $N_{I, c u t}^{(n)}=(n-1) 2^{n-1}$, the number of diagrams of type II turns out to be $N_{I I, \text { cut }}^{(n)}=(n-2) 2^{n-2}$ and the number of diagrams of type III is $N_{I I I, \text { cut }}^{(n)}=(n-2)(n-3) 2^{n-3}$. With the total number of diagrams after the cut, $N_{\text {cut }}^{(n)}=[4(n-1)+(n-1)(n-2)] 2^{n-3}$, we get for the double diffractive cross section

$$
\frac{d \sigma_{D D}\left(Y, Y_{0}, Y_{1}\right)}{d Y_{0} d Y_{1}}=\alpha^{2} e^{-\alpha Y_{0}-\alpha Y_{1}} \sum_{n=2}^{\infty}(-1)^{n} n ! x^{n} N_{c u t}^{(n)} .
$$

In high energy limit, while $Y_{0}$ and $Y_{1}$ large, one obtains

$$
\frac{d \sigma_{D D}\left(Y, Y_{0}, Y_{1}\right)}{d Y_{0} d Y_{1}} \simeq \frac{1}{4} \alpha^{2} e^{-\alpha Y_{0}-\alpha Y_{1}}
$$

or, equivalently,

$$
M_{x}^{2} M_{x}^{\prime 2} \frac{d \sigma_{D D}\left(s, M_{x}^{2}, M_{x}^{\prime 2}\right)}{d M_{x}^{2} d M_{x}^{\prime 2}} \simeq \frac{1}{4} \alpha^{2}\left(\frac{m_{p}^{2}}{M_{x}^{2}}\right)^{\alpha}\left(\frac{m_{p}^{2}}{M_{x}^{\prime 2}}\right)^{\alpha}
$$

The double diffractive cross section equals the product of two single diffractive cross sections at very large $Y$, which is in line with the naive expectation $d \sigma_{D D} / d Y_{0} d Y_{1} \sim$ $\left[d \sigma_{S D} / d Y_{0} d \sigma_{S D} / d Y_{1}\right] / \sigma_{t o t}$ since the double diffractive process can be viewed as two separate and independent single diffractive processes occurring at both, the projectile and the target. Therefore one obtains a stronger $\alpha$ and diffractive mass suppression for the differential double diffractive cross section as compared with the differential single diffractive cross section. Also the double diffractive cross section unitarizes due to the multiple pomeron exchanges, instead of showing a rapid growth, when $Y$ becomes very large large.

\section{CENTRAL DIFFRACTIVE SCATTERING}

The central diffractive scattering, $p+p \rightarrow p+X+p$ (see Fig. 1C), as shown in Fig. 5 (higher order graph), is obtained by following the same procedure as in the previous sections. The un-cut amplitude of $k$-th order (i.e., $k$ pomeron exchanges between 0 and $Y$ in addition to the lowest order diagram) reads

$$
T_{k}^{(1)}\left(Y, Y_{1}, Y_{0}\right) \simeq(-1)^{k+2} 2 \alpha^{2} \alpha_{s}^{2}(k+1)(k+2) ! x^{k+2} e^{-\alpha Y_{1}+\alpha Y_{0}} .
$$




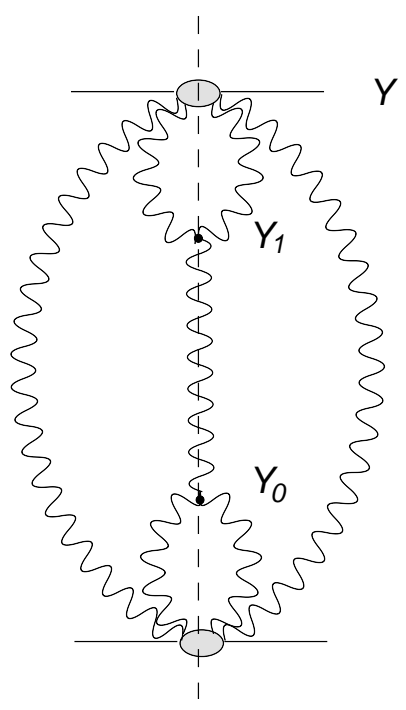

FIG. 5: Diffractive cut of general multi-pomeron exchange graphs which generate central diffractive dissociation.

When a diffractive cut is made, the diffractive cross section becomes

$$
\frac{d \sigma_{C D}\left(Y, Y_{0}, Y_{1}\right)}{d Y_{0} d Y_{1}} \simeq \alpha^{2} \alpha_{s}^{2} e^{-\alpha\left(Y_{1}-Y_{0}\right)} \sum_{n=2}^{\infty}(-1)^{n}(n-1) n ! x^{n} 2^{n},
$$

and for high energy scattering but $Y_{1}-Y_{0}=\delta Y=\ln \left(\frac{M_{X}^{2}}{m_{p}^{2}}\right)$ large $\left(M_{X}\right.$ is the central diffractive mass) it reduces to

$$
\frac{d \sigma_{C D}\left(Y, Y_{0}, Y_{1}\right)}{d Y_{0} d Y_{1}} \simeq \alpha^{2} \alpha_{s}^{2} e^{-\alpha \delta Y}=\alpha^{2} \alpha_{s}^{2}\left(\frac{m_{p}^{2}}{M_{x}^{2}}\right)^{\alpha} .
$$

The suppression $\alpha^{2} \alpha_{s}^{2}$ can be easily understood by looking at the lowest order graph (Fig. 5 without the two pomerons on both sides of length $Y$ ): The merging at $Y_{0}$ gives an $\beta$ and the subsequent splitting at $Y_{1}$ a $\alpha$. The rapidity dependence is $\exp [\alpha Y] \exp \left[\alpha\left(Y-\left(Y_{1}-Y_{0}\right)\right)\right]$. The remaining factor of $\alpha_{s}^{4}$ comes from the coupling of the pomerons to the scattering particles, thus, giving altogether $\alpha^{2} \alpha_{s}^{2} \exp \left[-\alpha\left(Y_{1}-Y_{0}\right)\right] x^{2}$, where $x=\alpha_{s}^{2} \exp [\alpha Y]$, which explains the result in (20). The higher order graphs obtained by adding further pomeron exchanges as shown in Fig. 5 lead to a $Y$-dependence in accordance with unitarity limits. The differential central diffractive cross section is strongly $\alpha_{s}$-suppressed $\left(\alpha \sim \alpha_{s}\right)$ as compared with the differential single (double) diffractive cross section.

In summary, we systematically calculate single diffractive, double diffractive and central diffractive cross sections within the zero transverse dimensional Regge model [1]. The resum- 
mation of all higher order dominant diagrams shows that diffractive cross sections gradually approach unitarity limits instead of increasing rapidly at very large energies. Moreover, we do find powers of $\alpha$ suppression in the inclusive diffractive cross sections.

Acknowledgements: We acknowledge numerous stimulating discussions with Alfred Mueller. A. Sh. acknowledges financial support by the Deutsche Forschungsgemeinschaft under contract Sh 92/2-1 and wishes to thank the theory group at DESY for hospitality during his visit when this work was being completed.

[1] A. I. Shoshi and B. W. Xiao, Phys. Rev. D 73094014 (2006).

[2] A. H. Mueller, A. I. Shoshi and S. M. H. Wong, Nucl. Phys. B 715440 (2005); E. Iancu and D. N. Triantafyllopoulos, Phys. Lett. B 610253 (2005); A. Kovner and M. Lublinsky, Phys. Rev. D 71085004 (2005); Y. Hatta, E. Iancu, L. McLerran, A. Stasto and D. N. Triantafyllopoulos, Nucl. Phys. A 764423 (2006).

[3] I. Balitsky, Nucl. Phys. B 46399 (1996); Phys. Rev. Lett. 812024 (1998); Phys. Lett. B 518 $235(2001)$.

[4] J. Jalilian-Marian, A. Kovner, A. Leonidov and H. Weigert, Nucl. Phys. B 504415 (1997); Phys. Rev. D 59014014 (1999). E. Iancu, A. Leonidov and L. D. McLerran, Phys. Lett. B 510133 (2001); Nucl. Phys. A 692583 (2001); H. Weigert, Nucl. Phys. A 703823 (2002).

[5] Y. V. Kovchegov, Phys. Rev. D 60034008 (1999); Phys. Rev. D 61074018 (2000).

[6] A. H. Mueller and A. I. Shoshi, Nucl. Phys. B 692175 (2004); E. Iancu, A. H. Mueller and S. Munier, Phys. Lett. B 606342 (2005).

[7] Y. Hatta, E. Iancu, C. Marquet, G. Soyez and D. N. Triantafyllopoulos, "Diffusive scaling and the high-energy limit of deep inelastic scattering in QCD at large N(c)," Nucl. Phys. A 773, 95 (2006).

[8] S. Munier and A. Shoshi, Phys. Rev. D 69074022 (2004).

[9] A. H. Mueller and G. P. Salam, Nucl. Phys. B 475293 (1996);

[10] P. Rembiesa and A. M. Stasto, Nucl. Phys. B 725251 (2005).

[11] M. Kozlov and E. Levin, Nucl. Phys. A 779, 142 (2006).

[12] M. Kozlov, E. Levin, V. Khachatryan and J. Miller, "The BFKL pomeron calculus in zero transverse dimensions: Diffractive processes and survival probability for central diffractive 
production," arXiv:hep-ph/0610084.

[13] J. P. Blaizot, E. Iancu and D. N. Triantafyllopoulos, "A zero-dimensional model for highenergy scattering in QCD," arXiv:hep-ph/0606253.

[14] S. Bondarenko, L. Motyka, A. H. Mueller, A. I. Shoshi and B. W. Xiao, "On the equivalence of Reggeon field theory in zero transverse dimensions and reaction-diffusion processes," arXiv:hep-ph/0609213.

[15] A. Kovner and M. Lublinsky, Nucl. Phys. A 767171 (2006).

[16] E. Iancu and D. N. Triantafyllopoulos, Nucl. Phys. A 756, 419 (2005).

[17] V. A. Abramovsky, V. N. Gribov and O. V. Kancheli, Yad. Fiz. 18 (1973) 595 [Sov. J. Nucl. Phys. 18308 (1974)].

[18] K. Goulianos, Phys. Lett. B 358(1995) 379 .

[19] A. H. Mueller, Eur. Phys. J. A 119 (1998);A. Hebecker, Phys. Rept. 3311 (2000).

[20] E. Gotsman, E. Levin and U. Maor, Phys. Lett. B 438229 (1998).

[21] J. Koplik and A. H. Mueller, Phys. Rev. D 123638 (1975); E. Levin, "An introduction to pomerons," arXiv:hep-ph/9808486.

[22] J. Bartels, M. Salvadore and G. P. Vacca, Eur. Phys. J. C 42, 53 (2005); J. Bartels and M. G. Ryskin, Z. Phys. C 76, 241 (1997).

[23] Y. V. Kovchegov and E. Levin, Nucl. Phys. B 577, 221 (2000); M. A. Braun, Phys. Lett. B 483, 105 (2000).

[24] D. Treleani, Int. J. Mod. Phys. A 11, 613 (1996). 